\title{
Fruquintinib with gefitinib as first-line therapy in patients carrying EGFR mutations with advanced non-small cell lung cancer: a single-arm, phase II study
}

\author{
Shun Lu ${ }^{1 \#}$, Jian-Ying Zhou ${ }^{2 \#}$, Xiao-Min Niu ${ }^{1}$, Jian-Ya Zhou ${ }^{2}$, Hong Jian ${ }^{1}$, Hong-Yan Yin ${ }^{3}$, Sha Guan ${ }^{3}$, \\ Lin-Fang Wang ${ }^{3}$, Ke $\mathrm{Li}^{3}$, James $\mathrm{He}^{3}$, Wei-Guo $\mathrm{Su}^{3}$ \\ ${ }^{1}$ Shanghai Lung Cancer Center, Shanghai Chest Hospital, Shanghai Jiao Tong University, Shanghai, China; ${ }^{2}$ The First Affiliated Hospital, College of \\ Medicine, Zhejiang University, Hangzhou, China; ${ }^{3}$ Hutchison MediPharma, Shanghai, China \\ Contributions: (I) Conceptualization and design: S Lu; (II) Administrative support: S Lu, HY Yin, S Guan, LF Wang, K Li, J He, WG Su; (III) \\ Provision of study materials or patients: S Lu, JY Zhou, XM Niu, JY Zhou, H Jian; (IV) Collection and assembly of data: S Lu, JY Zhou, XM Niu, JY \\ Zhou, H Jian; (V) Data analysis and interpretation: S Lu, HY Yin, S Guan, LF Wang, K Li, J He, WG Su; (VI) Manuscript writing: All authors; (VII) \\ Final approval of manuscript: All authors. \\ \#These authors contributed equally to this research, and are shared first authors of this manuscript. \\ Correspondence to: Dr. Shun Lu. Shanghai Lung Cancer Center, Shanghai Chest Hospital, Shanghai Jiao Tong University, 241 West Huaihai Road, \\ Shanghai 200030, China. Email: shunlu@sjtu.edu.cn.
}

Background: Fruquintinib is an oral vascular endothelial growth factor receptor inhibitor. Previous gefitinib studies with anti-angiogenics show promising efficacy. This phase II trial assessed efficacy and safety of fruquintinib in combination with gefitinib, in patients with advanced non-small cell lung cancer (NSCLC). Methods: Fifty patients with stage IIIB/IV NSCLC and an epidermal growth factor receptor (EGFR) exon19 deletion or exon-21 L858R mutation were enrolled between January 2017 and June 2019. Per protocol (version 1.0), patients received $4 \mathrm{mg}$ fruquintinib once daily (qd) Days 1-21 of Cycle 1, using a 3-week-on/1week-off schedule, plus continuous gefitinib $250 \mathrm{mg}$ qd. If tolerated, patients proceeded to fruquintinib $5 \mathrm{mg}$ qd (fruquintinib $5 \mathrm{mg}$ group, $\mathrm{n}=26$ ). Following protocol updates, dose escalation of fruquintinib from $4 \mathrm{mg}$ qd to $5 \mathrm{mg}$ qd was not allowed. The primary efficacy endpoint was objective response rate (ORR); secondary endpoints included progression-free survival (PFS), disease control rate (DCR), time to response, duration of response and adverse events (AEs).

Results: ORR was 73.5\% (95\% CI, 58.9-85.1) and DCR was 98.0\% (95\% CI, 89.2-100.0). Median PFS was 14.7 months for both groups; PFS was highest for patients with exon-19 deletion (16.5 months; $95 \%$ CI, 12.9-21.2). Grade $\geq 3$ treatment-emergent AEs occurred in 17 (65.3\%; fruquintinib $5 \mathrm{mg}$,) and 11 patients (45.8\%; $4 \mathrm{mg}$ ). Serious AEs were recorded for nine patients (fruquintinib $5 \mathrm{mg}$, six patients; $4 \mathrm{mg}$, three).

Conclusions: Fruquintinib and gefitinib treatment showed an acceptable safety profile and promising efficacy in patients with NSCLC.

Keywords: Non-small cell lung cancer (NSCLC); fruquintinib; gefitinib; epidermal growth factor receptor (EGFR); efficacy

Submitted Sep 27, 2020. Accepted for publication Dec 23, 2020.

doi: $10.21037 /$ tlcr-20-1028

View this article at: http://dx.doi.org/10.21037/tlcr-20-1028 


\section{Introduction}

Lung cancer is the most commonly diagnosed cancer, and the leading cause of cancer-related death globally (18.4\%) and in China $(24.1 \%)(1,2)$. In 2018, 774,323 new cases of lung cancer were diagnosed in China (2); while a 2015 report shows lung cancer deaths made up $6.2 \%$ of the country's total mortality (3). Non-small cell lung cancer (NSCLC) accounts for more than three-quarters of all lung cancers, and an estimated $70 \%$ of these patients have advanced disease (stage IIIB or IV) at first diagnosis (4).

The vascular endothelial growth factor (VEGF) signaling pathway, involved in angiogenesis and the development of malignant tumors, is a key target for cancer therapies (5). VEGF/ vascular endothelial growth factor receptor (VEGFR) inhibitors used for treatment of NSCLC are mainly used in combination with chemotherapeutic agents (6-9). Fruquintinib is an orally available VEGFR inhibitor that is highly selective for VEGFR-1, 2 and 3 (10). It has been approved in China for third-line treatment of patients with metastatic colorectal cancer, and phase II and III trials in NSCLC showed improved progression-free survival (PFS) compared to placebo $(11,12)$.

Epidermal growth factor receptor (EGFR) gene mutation is common in Chinese patients with NSCLC (45.1\%) and it is often used to identify patients that may benefit from targeted treatments (13). EGFR-tyrosine kinase inhibitors (TKIs) are the standard initial treatment for patients with EGFR mutation-positive NSCLC; gefitinib, an EGFRTKI, is widely used as monotherapy in China and globally. Clinical studies on gefitinib show better quality of life and PFS in advanced NSCLC patients with EGFR mutations, compared with chemotherapy $(14,15)$. However, treatment resistance to EGFR-TKIs is an emerging issue, primarily caused by an acquired EGFR T790M mutation. Other resistance mechanisms that have been reported include amplification of human epidermal growth factor receptor 2 (HER2) and/or mutation of phosphatidylinositol-4,5bisphosphate 3-kinase catalytic subunit alpha (PIK3CA) and/or B-Raf proto-oncogene (BRAF) (16). Approximately $50-65 \%$ of patients acquire resistance after receiving EGFR-TKI treatment (17). Third generation EGFRTKIs were originally developed for patients with the T790M acquired resistant mutation (18). Osimertinib, a third-generation EGFR-TKI, showed improved efficacy compared with pemetrexed plus platinum after progression of disease (PD) on a first-generation EGFR-TKI (19) and a lower percentage of grade $\geq 3$ adverse events (AEs) compared with first-generation EGFR-TKIs (20). Note, at the beginning of this trial there were no third-generation EGFR-TKIs approved in China for first-line EGFR mutation-positive NSCLC (e.g., almonertinib) (21).

Preclinical data suggest simultaneous inhibition of EGFR and VEGFR signaling pathways could abrogate primary or acquired resistance to EGFR-TKIs (22). In cellderived xenograft models expressing EGFR, administration of fruquintinib with gefitinib showed substantial anti-tumor activity compared to monotherapy (23).

Several clinical studies have been conducted using concurrent treatment with EGFR inhibitors and VEGF or VEGFR inhibitors in the treatment of patients with advanced NSCLC $(24,25)$. Dual therapy with erlotinib and bevacizumab (NEJ026 trial: PFS, 16.9 vs. 13.3 months in erlotinib monotherapy) (25) or erlotinib with ramucirumab in the RELAY trial (PFS, 19.4 vs. 12.4 months in erlotinib plus placebo) (26) as approved by the Food and Drug Administration (FDA) in May 2020 (27), have shown improved efficacy, demonstrating that simultaneous inhibition of EGFR and VEGF/VEGFR signaling pathways may have a synergistic effect in patients with EGFR-sensitive mutant NSCLC. However, the VEGF/ VEGFR inhibitors in these combinations are administered intravenously, which in practice is inconvenient for patients, highlighting the need for more practical treatment options. A dual oral approach, as was examined in this study, offers superior efficacy compared to monotherapy, is less susceptible to drug resistance and is more convenient than intravenous treatment options for patients.

This single-arm phase II study aimed to assess the efficacy, safety and tolerability of fruquintinib with gefitinib in EGFR-sensitive mutant, advanced non-squamous NSCLC, as a potential combination therapy which has potential to offer advantages in reduced susceptibility to drug resistance and convenient administration. We present the following article in accordance with the TREND reporting checklist (available at http://dx.doi.org/10.21037/ tlcr-20-1028).

\section{Methods}

\section{Patients}

Participant flow through the study is shown in Figure S1. All patients provided signed informed consent prior to enrollment. Patients aged 18-75 years with histology/ cytology-confirmed stage IIIB/IV non-squamous NSCLC, 
naïve to systematic treatment for advanced diseases, with the presence of $\geq 1$ measurable tumor lesion [in accordance with Response Evaluation Criteria in Solid Tumor (RECIST) 1.1], and an expected survival >12 weeks were enrolled. Positive identification with an EGFR-sensitive mutation (EGFR exon-19 deletion or exon-21 L858R mutation) was required. Key exclusion criteria included prior treatment with a TKI or a monoclonal antibody against VEGF, VEGFR or EGFR (see Table S1 for further details of inclusion and exclusion criteria).

The study was conducted in accordance with the Declaration of Helsinki (as revised in 2013) and Guidelines for Good Clinical Practice, independently approved by the ethics committees of each participating center (NCT02976116) and informed consent was taken from all the patients.

\section{Design and treatment}

This investigation was an open-label, single-arm, multicenter, phase II trial conducted at Shanghai Chest Hospital and the First Affiliated Hospital, College of Medicine, Zhejiang University, in China. There were three protocol amendments during the study; contributing factors included change in fruquintinib dose, change in study site, update to exclusion criteria, and extension of timeline. Consequently, the first 26 patients enrolled (per protocol version 1.0), received oral fruquintinib $4 \mathrm{mg}$ once daily (qd) from Days 1-21 of Cycle 1 (3-week-on/1-week-off) plus continuous oral gefitinib at a fixed dose of $250 \mathrm{mg}$ qd over the four weeks. Patients with no reported treatmentrelated grade $\geq 3$ AEs or grade $\geq 2$ liver AEs proceeded to further cycles with an increased fruquintinib dose $(5 \mathrm{mg} \mathrm{qd}$; fruquintinib $5 \mathrm{mg}$ group). The next 24 enrolled patients (per subsequent protocols) received oral fruquintinib $4 \mathrm{mg}$ qd from Days 1-21 of a 4-week cycle (3-week-on/1week-off), plus continuous oral gefitinib at a fixed dose of $250 \mathrm{mg}$ qd for all cycles (fruquintinib $4 \mathrm{mg}$ group). All enrolled patients received fruquintinib combined with gefitinib until withdrawal of informed consent, disease progression, intolerable toxicity or death. Tumors were evaluated (RECIST 1.1) once every 8 weeks. If evaluated as complete response (CR) or partial response (PR), this was re-confirmed 4 weeks later.

$\mathrm{AE}$ related dose interruption or reduction of fruquintinib, and/or dose interruption of gefitinib were permitted and recorded during the study. No dose reduction of gefitinib was allowed.
A biomarker exploratory informed consent form was collected according to ethical regulations of each participating hospital. Patients' blood samples were collected at baseline, and at PD. Patients' tumor samples were collected at baseline. DNA or circulating free DNA (cfDNA) was isolated according to manufacturer's protocol (Qiagen). Libraries were constructed using the KAPA Hyper DNA Library Prep Kit (KAPA Biosystems). Libraries were captured by probes for targeted sequencing cover exons and selected introns of 425 solid tumorrelated genes (Geneseeq PRIME panel). The library was sequenced on Illumina HiSeq 4000 Systems according to the manufacturer's instructions.

Blood samples to determine plasma concentration of fruquintinib were collected 10 minutes prior to administration on Day 2, 14 and 21 of Cycle 1, and for gefitinib, 10 minutes prior to administration on Days 21 and 28 of Cycle 1.

\section{Study endpoints}

The primary efficacy endpoint was objective response rate (ORR) (RECIST 1.1 criteria), defined as the percentage of patients with confirmed CR or PR. Secondary efficacy endpoints were (I) PFS, time from the first dose of investigational product to the first documentation of $\mathrm{PD}$ (RECIST 1.1 criteria) or death, whichever came first; (II) disease control rate (DCR), the percentage of patients with CR, PR, or stable disease (SD); (III) time to response (TTR), time from the first dose of investigational product to the first occurrence of CR or PR, and (IV) duration of response (DoR), time from the first occurrence of CR or PR to PD or death. Exploratory endpoints included correlation between biomarkers, related to EGFR or VEGFR signaling pathway in tissues and/or blood, and the corresponding therapeutic effect, and the steady-state plasma concentrations of fruquintinib and gefitinib.

Pharmacokinetics were presented as mean plasma concentrations of fruquintinib and gefitinib and their metabolites at each time point.

Safety assessments included treatment exposure, laboratory examinations, vital signs, electrocardiogram (ECG), echocardiogram, Eastern Cooperative Oncology Group performance status (ECOG PS), and AEs. AEs were coded using MedDRA version 22.0 and graded according to the National Cancer Institute Common Terminology Criteria for Adverse Events (NCI-CTCAE v4.03). Treatment-emergent AEs (TEAEs) were those occurring 
between the first administration of study treatment and within 33 days post-final treatment; serious AEs (SAEs) were judged by investigators to be related with the study treatment that occurred within 33 days after the end of study treatment.

\section{Statistical analyses}

Sample size was determined based on a predicted observed ORR in 40 patients. For $60 \%$ ORR the $95 \%$ confidence interval (CI) was 43.3-75.1, and for 70\% ORR it was 53.583.4. All statistical analyses were conducted using SAS 9.4 (SAS Institute). The full analysis set (FAS) was defined as all patients who received at least one dose of study treatment (fruquintinib or gefitinib). This analysis set was used for analysis of demographic and baseline characteristics, PFS, drug exposure and safety data. The efficacy evaluable analysis set (EEAS) was defined as all patients who received at least one dose of study treatment (fruquintinib or gefitinib) and had tumor evaluation at and post baseline. This set was used for analysis of ORR, DCR, TTR and other efficacy variables.

The $95 \%$ CI of the primary efficacy variable, ORR, and DCR were calculated using the Clopper-Pearson method. Median PFS, TTR and DoR were estimated using the Kaplan-Meier method.

Descriptive statistics were used for the plasma drug concentrations at different time points.

Subgroup analyses of efficacy variables, PD, PFS, and AEs, were performed by EGFR mutation status.

\section{Results}

\section{Patients and exposure}

Between January 5, 2017 and June 28, 2019 (cut-off date), 78 patients from Shanghai Chest Hospital and First Affiliated Hospital, College of Medicine, Zhejiang University were screened, of whom 50 were successfully enrolled to receive study treatment (FAS). One patient was not eligible for the EEAS $(n=49)$ due to no tumor evaluation after baseline. There was an even balance of male $(50.0 \%)$ and female patients $(50.0 \%)$ in the FAS with a mean age ( \pm standard deviation) of 57.5 years $( \pm 9.84)$, most being $<65$ years of age $(70.0 \%$; Table 1$)$. There were $92.0 \%$ of patients who were initially diagnosed with metastatic disease (Table 1). Approximately half the study population carried the EGFR exon-19 deletion (26 patients, 52.0\%) or the EGFR exon-21 L858R mutation (24 patients, 48.0\%). Overall, baseline characteristics were balanced between the fruquintinib 5 and $4 \mathrm{mg}$ treatment groups.

Median duration of exposure to fruquintinib was just over 1 year for all patients (fruquintinib $5 \mathrm{mg}, 12.8$ months; fruquintinib $4 \mathrm{mg}, 13.3$ months). The same was observed for gefitinib exposure (12.8 and 13.3 months, respectively). The mean ( \pm standard deviation) relative dose intensity was $0.78( \pm 0.18)$ versus $0.82( \pm 0.15)$ for fruquintinib, and 0.96 $( \pm 0.08)$ versus $0.97( \pm 0.04)$ for gefitinib in the respective treatment groups (Table S2).

\section{Efficacy}

Of the 49 patients (EEAS), 36 (73.5\%) had PR, 12 (24.5\%) had SD, and one patient (2.0\%) had PD (Table 2). Overall ORR was $73.5 \%$ (95\% CI, 58.9-85.1) of patients for fruquintinib combined with gefitinib; comparable rates were seen in both treatment groups [fruquintinib $5 \mathrm{mg}, 73.1 \%$, (95\% CI, 52.2-88.4); fruquintinib $4 \mathrm{mg}, 73.9 \%$, (95\% CI, 51.6-89.8); Table 2].

Median PFS (FAS) was 14.7 months (95\% CI, 12.521.2), with no difference observed between the fruquintinib $5 \mathrm{mg}$ and $4 \mathrm{mg}$ groups (14.7 months; Figure 1).

Estimated 1-year PFS rates (FAS) were $72.1 \%$ (fruquintinib $5 \mathrm{mg}$, 95\% CI, 48.0-86.4) and $65.5 \%$ (fruquintinib $4 \mathrm{mg}$, 95\% CI, 40.8-81.8). DCR in the EEAS was $98.0 \%$ (95\% CI, 89.2-100.0). DCR achieved in the fruquintinib $5 \mathrm{mg}$ group (100.0\%, 95\% CI, 86.8-100.0) was slightly higher than in the $4 \mathrm{mg}$ group (95.7\%, 95\% CI, 78.1-99.9; Table 2). The median TTR (EEAS) was 1.81 and 1.84 months for the fruquintinib 5 and $4 \mathrm{mg}$ groups, respectively (Figure 2).

Overall median DoR was 12.9 months; 12.9 months (95\% CI, 8.3-19.4) for fruquintinib $5 \mathrm{mg}$ group and 13.0 months (95\% CI, 9.0-NA) for fruquintinib $4 \mathrm{mg}$ group (Figure 3).

Subgroup analysis revealed patients with the EGFR exon-21 L858R mutation $(n=24)$ had median PFS of 14.7 months (95\% CI, 10.1-NA), while those with the EGFR exon-19 deletion $(n=26)$ had median PFS of 16.5 months (95\% CI, 12.9-21.2).

\section{Safety}

All $50(100.0 \%)$ patients in the FAS experienced at least one TEAE. The most common were elevated aspartate aminotransferase (AST) in 42 patients (84.0\%), elevated alanine aminotransferase (ALT) in 41 patients $(82.0 \%)$, 
Table 1 Summary of demographic data and baseline characteristics-FAS

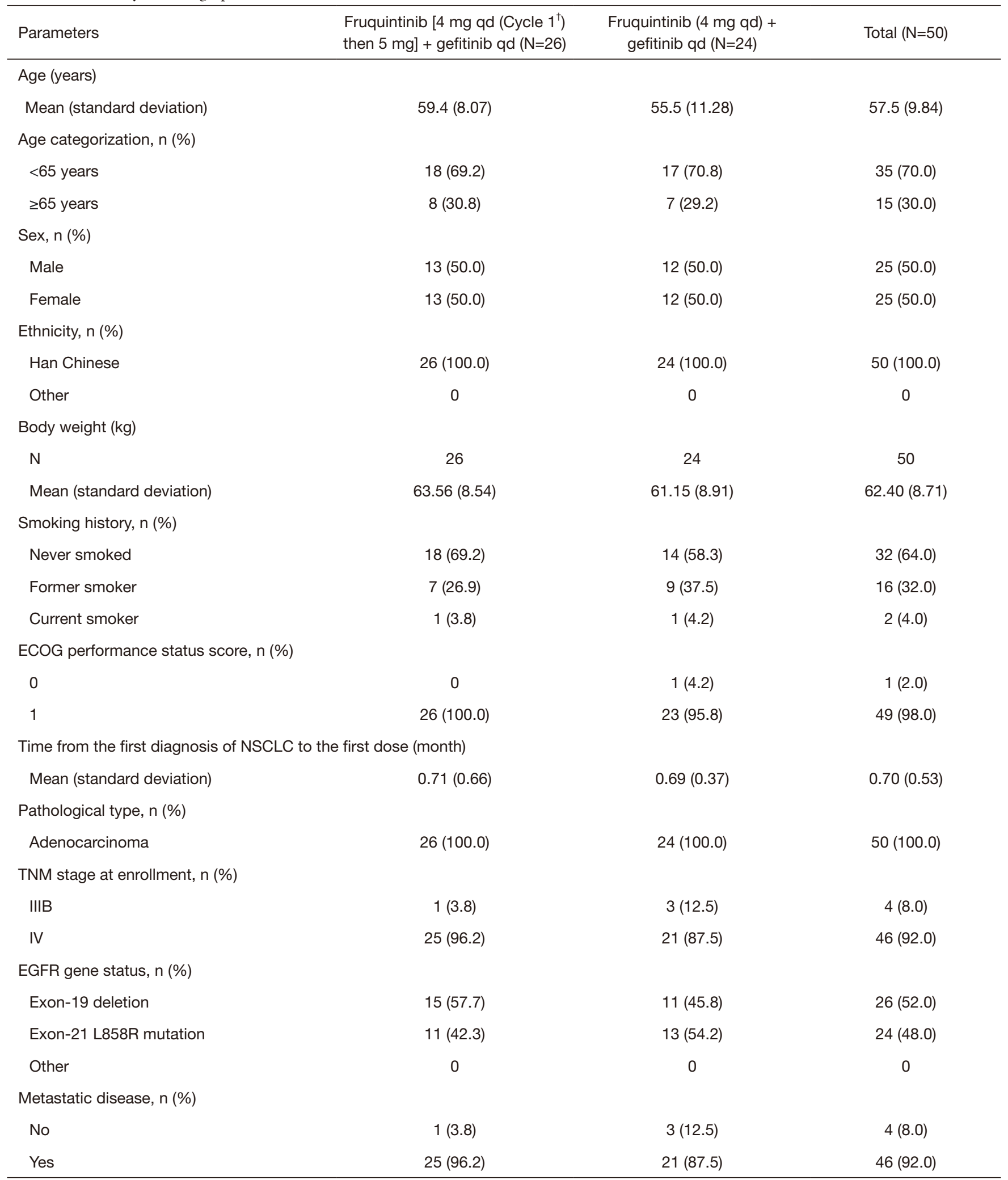

${ }^{\dagger}$, Cycle 1 was 4 weeks duration. FAS, full analysis set; ECOG, Eastern Cooperative Oncology Group; EGFR, epidermal growth factor receptor. 
Table 2 Analysis of objective response rate and disease control rate-EEAS

\begin{tabular}{|c|c|c|c|}
\hline Parameters & $\begin{array}{c}\text { Fruquintinib [ } 4 \mathrm{mg} \text { qd }\left(\text { Cycle } 1^{\dagger}\right) \text { then } 5 \\
\mathrm{mg}]+ \text { gefitinib } 250 \mathrm{mg} \mathrm{qd}(\mathrm{N}=26)\end{array}$ & $\begin{array}{l}\text { Fruquintinib (4 mg qd) + gefitinib } 250 \mathrm{mg} \\
\qquad \mathrm{qd}(\mathrm{N}=23)\end{array}$ & Total $(\mathrm{N}=49)$ \\
\hline \multicolumn{4}{|c|}{ The best response ${ }^{\ddagger}$} \\
\hline PR (\%) & $19(73.1)$ & $17(73.9)$ & $36(73.5)$ \\
\hline SD (\%) & 7 (26.9) & $5(21.7)$ & $12(24.5)$ \\
\hline NE & 0 & 0 & 0 \\
\hline $\mathrm{ORR}^{\S}$ & $19(73.1)$ & $17(73.9)$ & $36(73.5)$ \\
\hline $95 \% \mathrm{Cl}^{\Uparrow}$ & (52.21-88.43) & (51.59-89.77) & (58.92-85.05) \\
\hline
\end{tabular}

${ }^{\dagger}$, Cycle 1 was 4 weeks duration; ${ }^{\ddagger}$, those with complete response (CR) and partial response (PR) in the first evaluation needed to be confirmed after 4 weeks [Response Evaluation Criteria in Solid Tumor (RECIST) 1.1]; ${ }^{\S}$, objective response rate (ORR) = CR + PR; " , 95\% confidence interval $(\mathrm{Cl})$ was based on the Clopper-Pearson exact $\mathrm{Cl} ;{ }^{\dagger+}$, disease control rate $(\mathrm{DCR})=\mathrm{CR}+\mathrm{PR}+\mathrm{stable}$ disease $(\mathrm{SD})$. EEAS, efficacy evaluable analysis set; NE, not evaluable; PD, progression of disease.

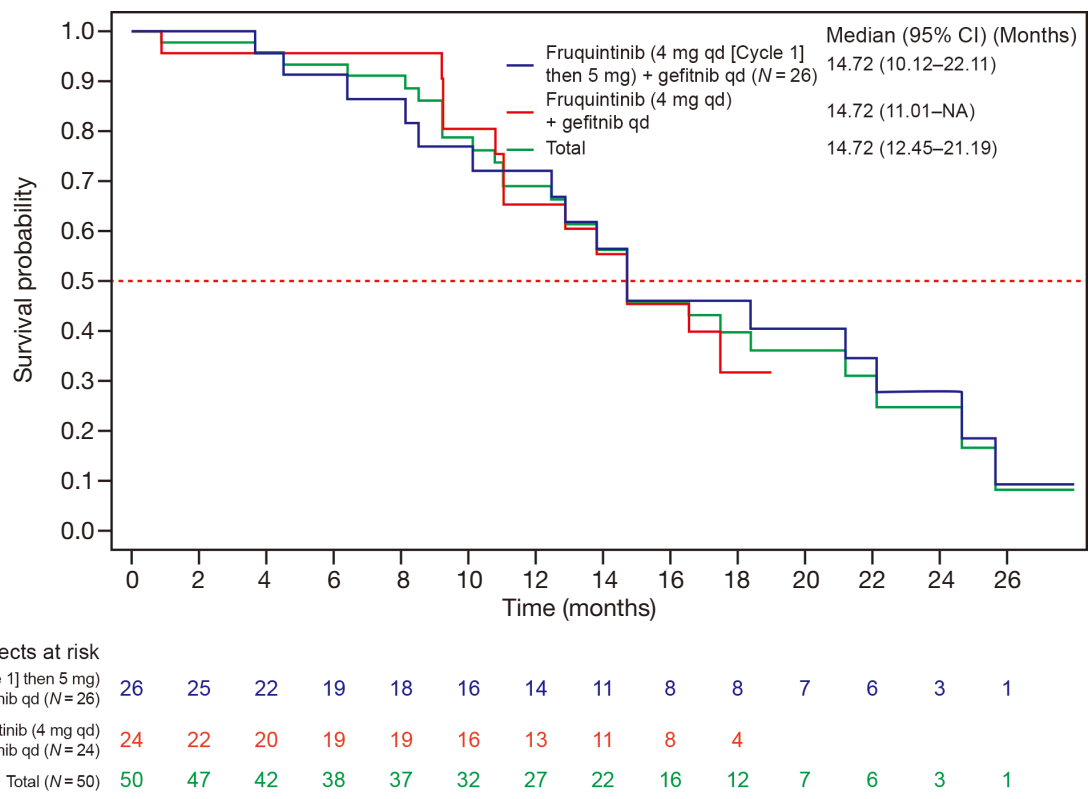

Figure 1 Kaplan-Meier plot of progression-free survival-FAS. The therapeutic effect of fruquintinib [4 mg qd (Cycle 1) then $5 \mathrm{mg}]+$ gefitinib $250 \mathrm{mg}$ qd versus fruquintinib (4 mg qd) + gefitinib $250 \mathrm{mg}$ qd determined by the stratified/unstratified Cox proportional risk model. Significance was determined by stratified/unstratified log-rank tests. FAS, full analysis set.

and proteinuria in 38 patients $(76.0 \%$; Table 3$)$. TEAEs were recorded in 24 and 26 patients in the fruquintinib 5 and $4 \mathrm{mg}$ treatment groups, respectively; 17 and 11 patients experienced one or more CTCAE grade $\geq 3$ TEAE respectively (Table 3).

More than half of all patients (FAS) experienced TEAEs leading to dose adjustment (including dose interruption and reduction) of fruquintinib [fruquintinib $5 \mathrm{mg}, 20$ 

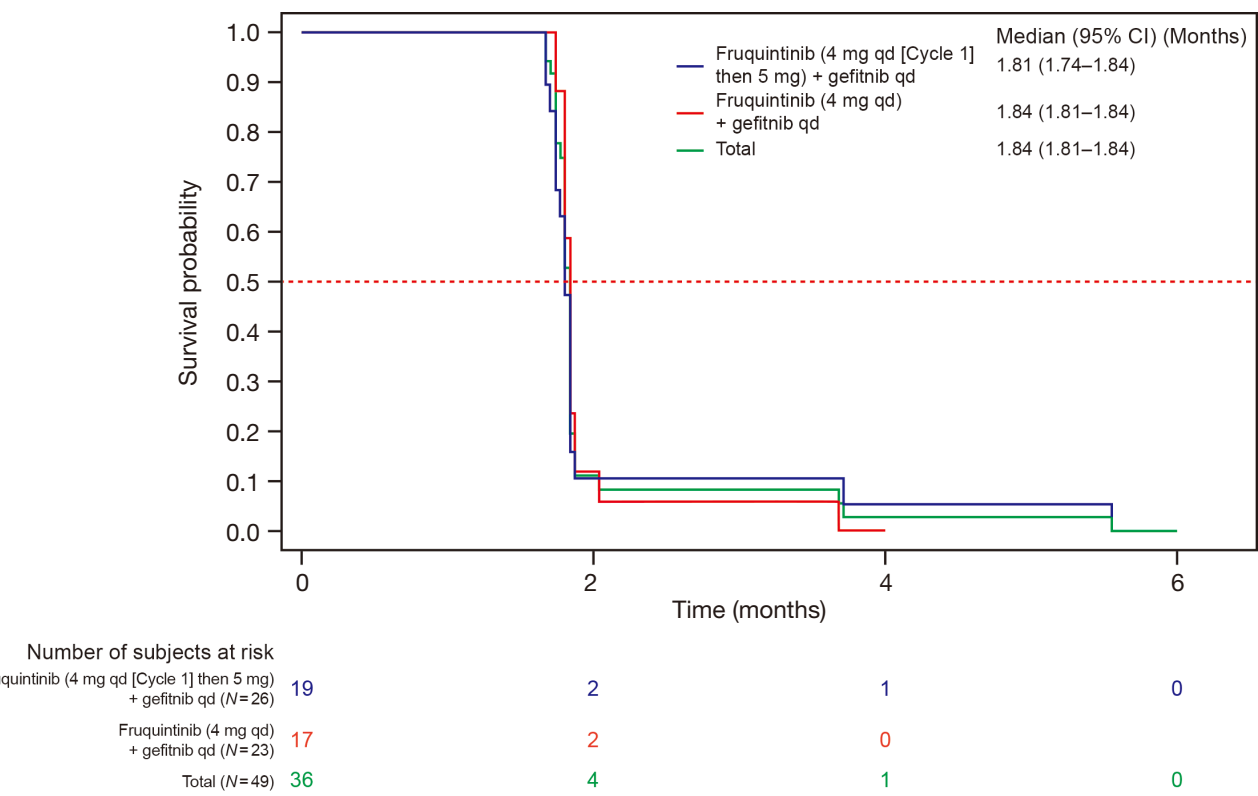

0

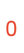

0

Figure 2 Kaplan-Meier plot of time to response-EEAS. The therapeutic effect of fruquintinib [4 mg qd (Cycle 1) then $5 \mathrm{mg}]+$ gefitinib $250 \mathrm{mg}$ qd versus fruquintinib (4 mg qd) + gefitinib $250 \mathrm{mg}$ qd determined by the stratified/unstratified Cox proportional risk model. Significance was determined by stratified/unstratified log-rank tests. EEAS, efficacy evaluable analysis set.

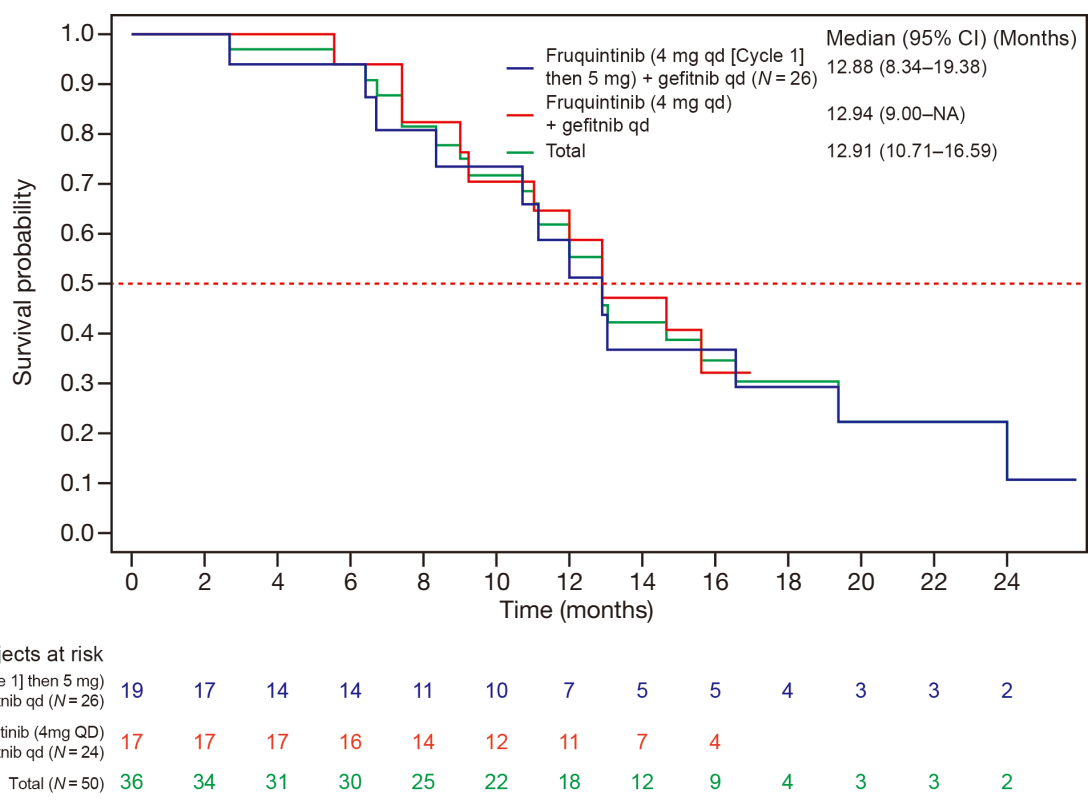

Figure 3 Kaplan-Meier plot of duration of response-EEAS. The therapeutic effect of fruquintinib [4 mg qd (Cycle 1) then $5 \mathrm{mg}]+$ gefitinib $250 \mathrm{mg}$ qd versus fruquintinib (4 mg qd) + gefitinib $250 \mathrm{mg}$ qd determined by the stratified/unstratified Cox proportional risk model. Significance was determined by stratified/unstratified log-rank tests. EEAS, efficacy evaluable analysis set. 
Table 3 Summary of the TEAE $^{\dagger}$ during study treatment-FAS

\begin{tabular}{|c|c|c|c|c|c|c|}
\hline \multirow[t]{2}{*}{ System organ class/preferred term } & \multicolumn{2}{|c|}{$\begin{array}{l}\text { Fruquintinib [4 mg qd }\left(\mathrm{Cycle}^{\ddagger}\right) \\
\text { then } 5 \mathrm{mg} \text { + gefitinib qd } \\
(\mathrm{N}=26), \mathrm{n}(\%)\end{array}$} & \multicolumn{2}{|c|}{$\begin{array}{c}\text { Fruquintinib (4 mg qd)+ } \\
\text { gefitinib } 250 \mathrm{mg} \text { qd }(\mathrm{N}=24), \\
\mathrm{n}(\%)\end{array}$} & \multicolumn{2}{|c|}{ Total (N=50), n (\%) } \\
\hline & All grades & Grade $\geq 3$ & All grades & Grade $\geq 3$ & All grades & Grade $\geq 3$ \\
\hline At least one adverse event & $26(100.0)$ & $17(65.4)$ & $24(100.0)$ & $11(45.8)$ & $50(100.0)$ & $28(56.0)$ \\
\hline Investigations & $26(100.0)$ & $11(42.3)$ & $24(100.0)$ & $3(12.5)$ & $50(100.0)$ & $14(28.0)$ \\
\hline Thyroid stimulating hormone increased & $23(88.5)$ & 0 & $14(58.3)$ & 0 & $37(74.0)$ & 0 \\
\hline Conjugated bilirubin increased & $14(53.8)$ & $1(3.8)$ & $8(33.3)$ & $1(4.2)$ & $22(44.0)$ & $2(4.0)$ \\
\hline Electrocardiogram QT prolonged & $8(30.8)$ & $1(3.8)$ & $9(37.5)$ & 0 & $17(34.0)$ & $1(2.0)$ \\
\hline Blood total bilirubin increased & $10(38.5)$ & 0 & $5(20.8)$ & 0 & $15(30.0)$ & 0 \\
\hline Electrocardiogram ST segment abnormality & $4(15.4)$ & 0 & $6(25.0)$ & 0 & $10(20.0)$ & 0 \\
\hline Electrocardiogram ST-T segment change & $6(23.1)$ & 0 & $4(16.7)$ & 0 & $10(20.0)$ & 0 \\
\hline Blood glucose increased & $6(23.1)$ & 0 & $4(16.7)$ & 0 & $10(20.0)$ & 0 \\
\hline Neutrophil decreased & $1(3.8)$ & 0 & $4(16.7)$ & 0 & $5(10.0)$ & 0 \\
\hline White blood cell decreased & $3(11.5)$ & 0 & $2(8.3)$ & 0 & $5(10.0)$ & 0 \\
\hline Platelets decreased & $2(7.7)$ & 0 & $3(12.5)$ & 0 & $5(10.0)$ & 0 \\
\hline Blood pressure increased & $4(15.4)$ & $1(3.8)$ & 0 & 0 & $4(8.0)$ & $1(2.0)$ \\
\hline Positive occult blood & $2(7.7)$ & 0 & $2(8.3)$ & 0 & $4(8.0)$ & 0 \\
\hline Electrocardiogram ST segment elevation & $1(3.8)$ & 0 & 0 & 0 & $1(2.0)$ & 0 \\
\hline Electrocardiogram T-wave high tip & 0 & 0 & $1(4.2)$ & 0 & $1(2.0)$ & 0 \\
\hline Electrocardiogram large voltage & $1(3.8)$ & 0 & 0 & 0 & $1(2.0)$ & 0 \\
\hline Thyroid hormone increased & 0 & 0 & $1(4.2)$ & 0 & $1(2.0)$ & 0 \\
\hline Blood urea nitrogen & $1(3.8)$ & 0 & 0 & 0 & $1(2.0)$ & 0 \\
\hline Renal and urinary disorders & $18(69.2)$ & $3(11.5)$ & $20(83.3)$ & $1(4.2)$ & $38(76.0)$ & $4(8.0)$ \\
\hline Proteinuria & $18(69.2)$ & $3(11.5)$ & $20(83.3)$ & $1(4.2)$ & $38(76.0)$ & $4(8.0)$ \\
\hline Hematuria & $2(7.7)$ & 0 & $3(12.5)$ & 0 & $5(10.0)$ & 0 \\
\hline Metabolism and nutrition disorders & $18(69.2)$ & $1(3.8)$ & $16(66.7)$ & $1(4.2)$ & $34(68.0)$ & $2(4.0)$ \\
\hline Hyperglycemia & $9(34.6)$ & 0 & $9(37.5)$ & 0 & $18(36.0)$ & 0 \\
\hline Hyponatremia & $7(26.9)$ & $1(3.8)$ & $3(12.5)$ & $1(4.2)$ & $10(20.0)$ & $2(4.0)$ \\
\hline Hypokalemia & $3(11.5)$ & 0 & $5(20.8)$ & 0 & $8(16.0)$ & 0 \\
\hline Hypomagnesemia & $4(15.4)$ & 0 & $3(12.5)$ & 0 & 7 (14.0) & 0 \\
\hline
\end{tabular}

Table 3 (continued) 
Table 3 (continued)

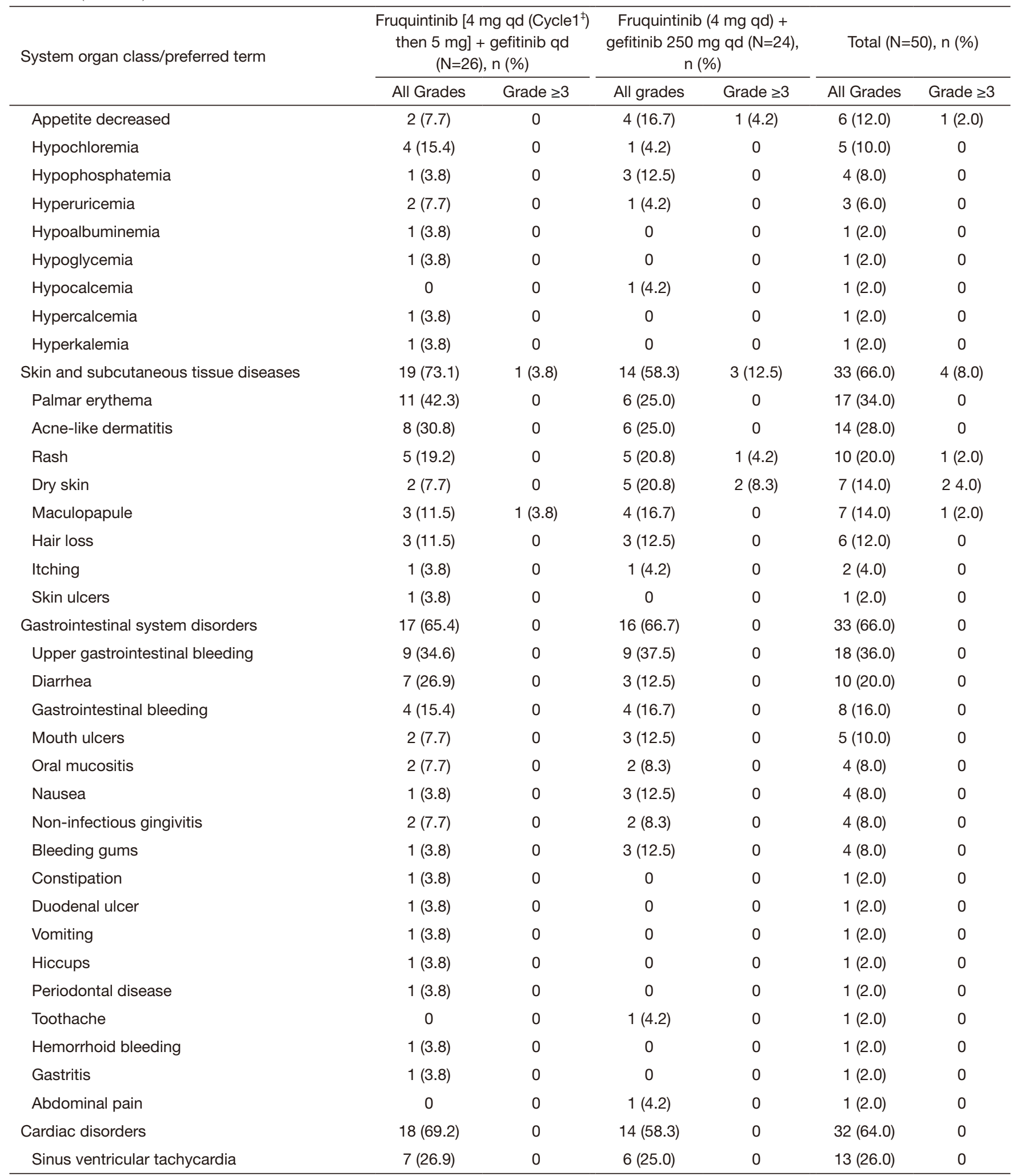

Table 3 (continued) 
Table 3 (continued)

\begin{tabular}{|c|c|c|c|c|c|c|}
\hline \multirow[t]{2}{*}{ System organ class/preferred term } & \multicolumn{2}{|c|}{$\begin{array}{l}\text { Fruquintinib [4 mg qd }\left(\mathrm{Cycle}^{\ddagger}\right) \\
\text { then } 5 \mathrm{mg}]+ \text { gefitinib qd } \\
(\mathrm{N}=26), \mathrm{n}(\%)\end{array}$} & \multicolumn{2}{|c|}{$\begin{array}{c}\text { Fruquintinib (4 mg qd) + } \\
\text { gefitinib } 250 \text { mg qd (N=24), } \\
n(\%)\end{array}$} & \multicolumn{2}{|c|}{ Total $(\mathrm{N}=50), \mathrm{n}(\%)$} \\
\hline & All grades & Grade $\geq 3$ & All grades & Grade $\geq 3$ & All grades & Grade $\geq 3$ \\
\hline Supraventricular extrasystoles & $7(26.9)$ & 0 & $4(16.7)$ & 0 & $11(22.0)$ & 0 \\
\hline Sinus bradycardia & $6(23.1)$ & 0 & $2(8.3)$ & 0 & $8(16.0)$ & 0 \\
\hline Ventricular arrhythmia & 0 & 0 & $1(4.2)$ & 0 & $1(2.0)$ & 0 \\
\hline Left bundle branch block & $1(3.8)$ & 0 & 0 & 0 & $1(2.0)$ & 0 \\
\hline Atrioventricular block & $1(3.8)$ & 0 & 0 & 0 & $1(2.0)$ & 0 \\
\hline Atrial tachycardia & 0 & 0 & $1(4.2)$ & 0 & $1(2.0)$ & 0 \\
\hline Cough & $5(19.2)$ & 0 & $2(8.3)$ & 0 & $7(14.0)$ & 0 \\
\hline Epistaxis & $3(11.5)$ & 0 & $2(8.3)$ & 0 & $5(10.0)$ & 0 \\
\hline Pneumothorax & $2(7.7)$ & 0 & $1(4.2)$ & $1(4.2)$ & $3(6.0)$ & $1(2.0)$ \\
\hline Cough up mucus & $1(3.8)$ & 0 & $2(8.3)$ & 0 & $3(6.0)$ & 0 \\
\hline Chest fluid buildup & $3(11.5)$ & 0 & 0 & 0 & $3(6.0)$ & 0 \\
\hline Lung inflammation & 0 & 0 & $2(8.3)$ & 0 & $24.0)$ & 0 \\
\hline Breath difficulty & 0 & 0 & $1(4.2)$ & 0 & $1(2.0)$ & 0 \\
\hline Hemoptysis & $1(3.8)$ & 0 & 0 & 0 & $1(2.0)$ & 0 \\
\hline Dry throat & $1(3.8)$ & 0 & 0 & 0 & $1(2.0)$ & 0 \\
\hline Endocrine disorders & $11(42.3)$ & 0 & $11(45.8)$ & 0 & $22(44.0)$ & 0 \\
\hline Hyperthyroidism & $8(30.8)$ & 0 & $10(41.7)$ & 0 & $18(36.0)$ & 0 \\
\hline Hypothyroidism & $6(23.1)$ & 0 & $2(8.3)$ & 0 & $8(16.0)$ & 0 \\
\hline Infections and infestations & $5(19.2)$ & $1(3.8)$ & $6(25.0)$ & $2(8.3)$ & $11(22.0)$ & $3(6.0)$ \\
\hline Lung infection & $3(11.5)$ & 0 & $2(8.3)$ & $1(4.2)$ & $5(10.0)$ & $1(2.0)$ \\
\hline Upper respiratory tract infection & 0 & 0 & $4(16.7)$ & $1(4.2)$ & $4(8.0)$ & $1(2.0)$ \\
\hline Paronychia & $2(7.7)$ & 0 & $2(8.3)$ & 0 & $4(8.0)$ & 0 \\
\hline Urinary tract infection & $1(3.8)$ & 0 & $1(4.2)$ & 0 & $2(4.0)$ & 0 \\
\hline Periodontitis & $1(3.8)$ & $1(3.8)$ & 0 & 0 & $1(2.0)$ & $1(2.0)$ \\
\hline Pharyngitis & 0 & 0 & $1(4.2)$ & 0 & $1(2.0)$ & 0 \\
\hline Infectious pneumonia & $1(3.8)$ & 0 & 0 & 0 & $1(2.0)$ & 0 \\
\hline Tinea pedis & 0 & 0 & $1(4.2)$ & 0 & $1(2.0)$ & 0 \\
\hline
\end{tabular}

Table 3 (continued) 
Table 3 (continued)

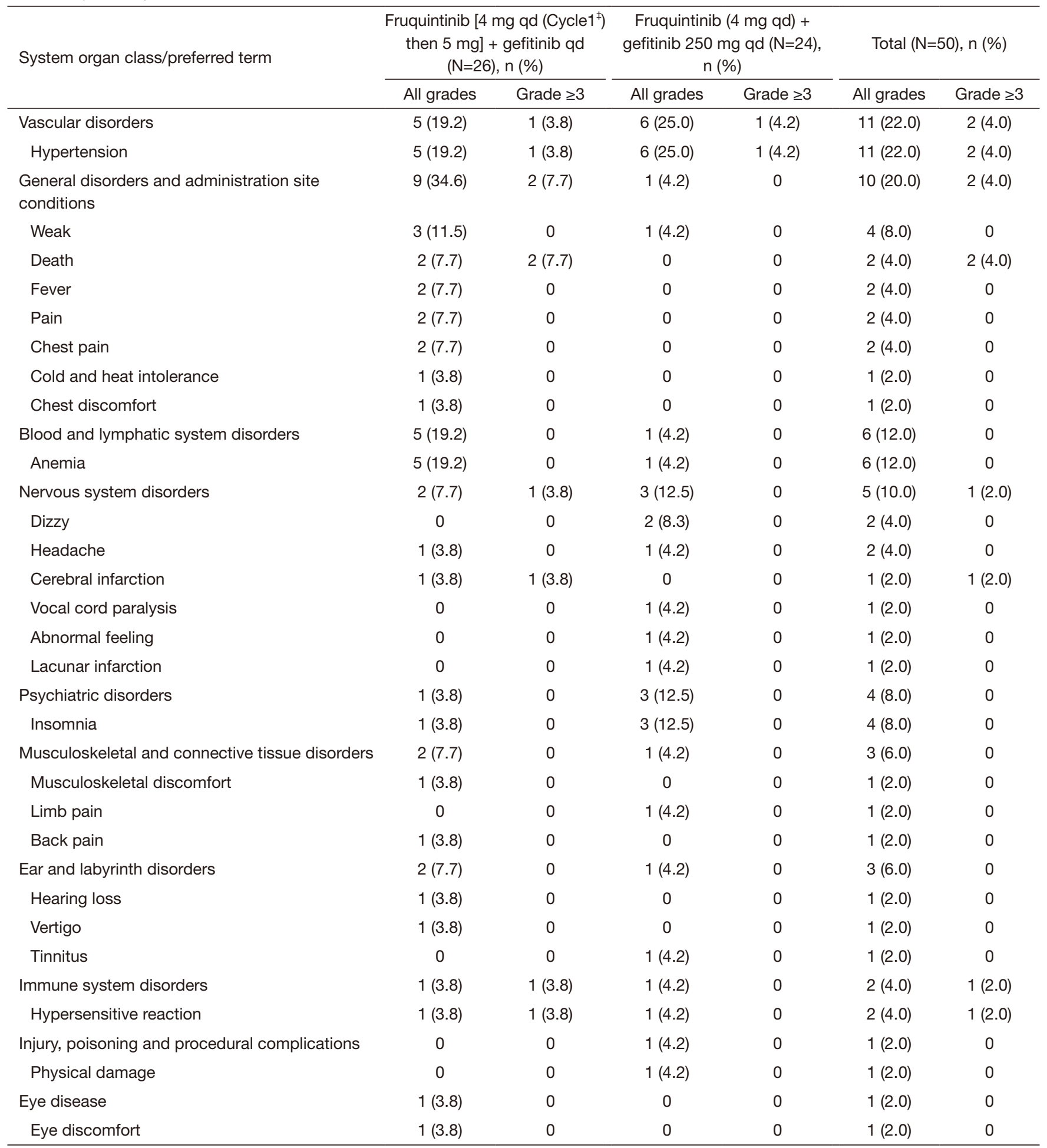

${ }^{\dagger}$, in case the same adverse event occurred for multiple times for the same patient, the event could only be counted once in calculation of it by the same system organ class and preferred term for the patient; ${ }^{\ddagger}$, Cycle 1 was 4 weeks duration; FAS, full analysis set; TEAE, treatment-emergent adverse event. 
patients (76.9\%); fruquintinib $4 \mathrm{mg}, 17$ patients $(70.8 \%)$ ] and gefitinib [fruquintinib $5 \mathrm{mg}, 15$ patients $(57.7 \%$ ); fruquintinib $4 \mathrm{mg}, 15$ patients $(62.5 \%)]$. Patients in the fruquintinib $5 \mathrm{mg}$ group experienced a higher number of TEAE that led to discontinuation of both fruquintinib and gefitinib than those in the fruquintinib $4 \mathrm{mg}$ group [fruquintinib; fruquintinib $5 \mathrm{mg}$ group, 6 patients (23.1\%); fruquintinib $4 \mathrm{mg}$ group, 4 patients $(16.7 \%)$; gefitinib, fruquintinib $5 \mathrm{mg}$ group, 6 patients (23.1\%); fruquintinib $4 \mathrm{mg}$ group, 4 patients (16.7\%); Table S3].

SAEs (Table S3) were recorded for nine patients (fruquintinib $5 \mathrm{mg}$, six patients; fruquintinib $4 \mathrm{mg}$, three patients), the most common being pleural effusion $(6.0 \%$ of patients, FAS) and pneumothorax (4.0\%). During the investigation, there were three patients in the fruquintinib $5 \mathrm{mg}$ group $(6.0 \%)$ who experienced an $\mathrm{AE}$ that led to death, which met the criteria of SAE that death within 30 days after end of treatment, and investigators concluded the three deaths as possibly unrelated to fruquintinib and gefitinib. One patient received treatment for 257 days, and died (AE term: acute cerebral infarction) 20 days after the last dose of study treatment; one patient received the treatment for 109 days, and died (AE term: death) from progression of disease 26 days after the last dose of study treatment; one patient received the treatment for 104 days, and died (AE term: death) of unknown cause 19 days after the last dose of study treatment.

All patients experienced at least one AE of special interest (fruquintinib $5 \mathrm{mg}, 26$ patients; fruquintinib $4 \mathrm{mg}$, 24 patients), the most common being hepatotoxicity $(90.0 \%)$, thyroid dysfunction $(88.0 \%)$, and proteinuria (76.0\%; Table S4).

\section{Biomarkers}

All enrolled patients were screened for an EGFR mutation at baseline; 17 patients volunteered their tumor tissue or plasma samples at baseline or after PD for biomarker exploratory. An EGFR-sensitive mutation was detected in $70 \%$ of baseline plasma samples. One patient, who had rapid PD after the first dose (26 days), was found to carry additional MET gene amplification at baseline both in tumor and plasma samples. The MET gene copy number was 16 in the tumor sample and 3.6 in the cfDNA sample performed separately by next-generation sequence (NGS) profiling (GENESEEQ PRIME PANEL). One patient showed KRAS amplification at baseline. For this patient, PR was the best response and median PFS was 9.4 months.
Ten patients volunteered their blood samples after PD. Among them, baseline EGFR genotyping was detected in two patients. EGFR (T790M), HER2, PIK3CA and BRAF resistance gene alterations were not present in these 10 patients.

\section{Pharmacokinetics}

The data for fruquintinib plasma concentrations after oral administration were available from 47 patients on Day 2 and 21 of Cycle 1, and from 48 patients on Day 14 of Cycle 1. The data for gefitinib concentration in plasma after oral administration were available from 47 patients on Day 21 of Cycle 1, and from 46 patients on Day 28 of Cycle 1. The geometric mean concentration of fruquintinib (4 $\mathrm{mg}$ group) was $71.6 \mathrm{ng} / \mathrm{mL}$ for Day 2, $156 \mathrm{ng} / \mathrm{mL}$ for Day 14 , and $159 \mathrm{ng} / \mathrm{mL}$ for Day 21 . The geometric mean concentration of gefitinib was $309 \mathrm{ng} / \mathrm{mL}$ for Day 21 and $313 \mathrm{ng} / \mathrm{mL}$ for Day 28 (Figure S2).

\section{Discussion}

This open-label, single-arm, phase II study demonstrated an overall ORR of $73.5 \%$ and DCR of $98.0 \%$ in Chinese patients with advanced EGFR mutation-positive nonsquamous NSCLC, treated with fruquintinib plus gefitinib. Efficacy data were similar between the fruquintinib 5 and $4 \mathrm{mg}$ groups.

The ORR and DCR achieved in this study is similar to that shown in a phase II clinical trial in EGFR-sensitive mutant, stage IIIB/IV NSCLC patients in Japan, treated with gefitinib (oral) and bevacizumab (intravenous; ORR 73.8\%; DCR 97.6\%) (28). The high DCR's likely reflect the prolonged PFS in both studies, which was approximately 15 months; however fruquintinib plus gefitinib consistently showed improved estimated 1-year PFS rate at $72.1 \%$ (95\% CI, 48.0-86.4, fruquintinib $5 \mathrm{mg}$ group) and $65.5 \%$ (95\% CI, 40.8-81.8, fruquintinib $4 \mathrm{mg}$ group) compared to the gefitinib plus bevacizumab combination therapy (1-year PFS rate $56.7 \%$; 95\% CI, 39.9-70.5) (28). The improved estimated 1-year PFS rate of fruquintinib plus gefitinib indicates higher rates of sustained response to combination therapy over a period of a year. Median PFS in this study (14.72 months) was approximately $30 \%$ higher than that reported for gefitinib monotherapy (10.4 months); estimated 1-year PFS rate was also notably higher (72.1\% vs. $42.1 \%$ ), indicating higher efficacy of fruquintinib plus gefitinib compared to gefitinib monotherapy (29). 
In the RELAY trial, the median PFS of ramucirumab plus erlotinib reached 19.4 months, which is numerically longer than that in this study (26). In a nationwide realworld study, researchers pointed out that the OS benefit for erlotinib users was superior, compared to gefitinib, and was similar to afatinib, a second-generation EGFR TKI $(30,31)$. Another real-word study suggested that second-generation EGFR TKIs are a reasonable upfront treatment choice due to the associated survival benefit (32). This potential efficacy difference between first-generation TKIs, or firstand second-generation TKIs, might led to the efficacy difference in their corresponding combination therapies. Besides, efficacy outcomes associated with EGFR TKIs varying amongst different ethnic subgroups were identified, suggesting that there may be an impact on efficacy in different populations from combination studies $(26,30,31)$.

Currently, the distinction in treatment efficacy outcomes for NSCLC patients with EGFR exon-19 deletion and EGFR exon-21 L858R mutations is not aligned across studies. In our study, median PFS was slightly higher in patients with EGFR exon-19 deletion than those with exon 21 L858R mutations (16.5 vs. 14.7 months) upon treatment with fruquintinib and gefitinib. Patel et al. found the median PFS was significantly longer for patients with exon-21 deleted than for those with positive exon21 (L858R) mutation (16 vs. 8 months) (33). However, Li et al. compared the two groups with a larger sample size and found no significant differences in median PFS (34). Subgroup analyses from a phase II study by Kim et al. indicate ORR and DCR were higher in patients with exon-19 deletions than L858R mutations, and suggest that patients with the exon-19 deletion may benefit more from gefitinib treatment (35). Further investigation for fruquintinib plus gefitinib treatment is needed to identify a possible superior treatment benefit for those with the exon19 deletion.

The administration of fruquintinib and gefitinib did not identify any new safety signals and appeared to be well tolerated in this population of patients with NSCLC. Increased ALT and AST are among the commonly reported AEs for gefitinib monotherapy (36), consistent with that observed in this study (elevated ALT, 41 patients; elevated AST, 42 patients). IPASS, a phase III randomized study on gefitinib compared to carboplatin/paclitaxel, showed that the incidence of elevated ALT (18.5\%) and AST (11.4\%) for gefitinib were lower than that observed with the combination treatment in this study $(84.0 \%$ and $82.0 \%$ of patients, respectively) (37). It would appear that the incidence of elevated transaminases are higher for fruquintinib plus gefitinib than for gefitinib monotherapy, which may reflect overlapping toxicities, the effects of which can be minimized by dose adjustment. Although there was no marked difference in drug exposure between the fruquintinib 5 and $4 \mathrm{mg}$ groups, the incidence of SAEs was $23.1 \%$ vs. $12.5 \%$, respectively. Incidence of grade $\geq 3$ AEs for fruquintinib 5 and $4 \mathrm{mg}$ was $65.4 \%$ vs. $45.8 \%$, respectively. These safety findings suggest fruquintinib $4 \mathrm{mg}$ is a better tolerated dose.

In this study one patient was found to carry MET amplification at baseline. This patient progressed quickly after the first dose, suggesting that MET amplification may accelerate tumor progression in patients with advanced EGFR mutation-positive NSCLC treated with the combination of fruquintinib and gefitinib, and a different combination treatment would need to be developed for patients with additional MET amplification.

There are varying frequencies of EGFR T790Macquired mutation reported with EGFR-TKI plus VEGFR inhibitor therapy $(26,38)$. The RELAY study reported comparable rates of T790M mutations in patients receiving monotherapy and combination therapy (47.0\% vs. $43.0 \%$ ) (26). A real-world study has reported bevacizumab plus EGFR-TKI group had lower T790M rates (38). In our study, T790M mutation was not found in the 10 cfDNA samples collected at PD. Other EGFRTKI resistance mechanisms such as HER2 amplification or PIK3CA and BRAF mutations were also not observed. Detection methods can report varying detection frequencies of T790M (39) and sensitivity in detecting. T790M in plasma cfDNA has been observed to be lower than that of tissue samples (40). Further investigation in larger sample groups is needed to confirm negative plasma-based results with tissue samples.

Full conclusions on the efficacy of treatment cannot be made on the basis of this single-arm study, nor can the data be extrapolated to a wider ethnic population; however, these preliminary findings can be used to inform future studies. On consideration of the efficacy and safety data from this study, the risk-benefit balance remains favorable for continuing further studies on this oral combination therapy. There appears to be increasing evidence that indicates third-generation EGFR-TKIs are more efficacious in the first-line treatment of patients with EGFR mutationpositive advanced NSCLC $(41,42)$. PFS for osimertinib compared to standard EGFR-TKIs is prolonged (18.9 vs. 10.2 months) (41); thus, it may be of interest to explore 
fruquintinib containing combination therapies containing third-generation EGFR-TKIs.

\section{Conclusions}

Combined fruquintinib and gefitinib treatment showed an acceptable safety profile and promising efficacy in newly diagnosed NSCLC patients harboring EGFR-activating mutations. The lower dose of fruquintinib (4 mg) in combination with gefitinib $(250 \mathrm{mg})$ was better tolerated, without compromising efficacy. Confirmatory studies with fruquintinib $(4 \mathrm{mg})$ in combination with gefitinib are required to further verify the full potential of this treatment.

\section{Acknowledgments}

We are thankful for all patients and their families as well as investigators in this study. Editorial assistance was provided by Anita Abeygoonesekera, from Meditech Media (Shanghai), which was funded by Hutchison MediPharma, in accordance with Good Publication Practice 3 guidelines.

Funding: This work was supported by Hutchison MediPharma (Shanghai); National Key R\&D Program of China [2016YFC1303300]; and Shanghai Science and Technology Innovation Program [19411950500].

\section{Footnote}

Reporting Checklist: The authors have completed the TREND reporting checklist. Available at http://dx.doi. org/10.21037/tlcr-20-1028

Data Sharing Statement: Available at http://dx.doi. org/10.21037/tlcr-20-1028

Conflicts of Interest: All authors have completed the ICMJE uniform disclosure form (available at http:// dx.doi.org/10.21037/tlcr-20-1028). SL reports grants from Hutchison MediPharma, grants from National Key R\&D Program of China, grants from Shanghai Science and Technology Innovation Program during the conduct of the study; other research support from AstraZeneca; Boehringer Ingelheim, Hutchison MediPharma and Roche; other speaker fees from AstraZeneca, Eli Lilly, Roche, and Sanofi; an advisor for AstraZeneca, Boehringer Ingelheim, Hutchison MediPharma and Roche outside the submitted work. HYY, SG, LFW, KL and JH report personal fees from Hutchison MediPharma during the conduct of the study. WGS reports personal fees from Hutchison MediPharma and Hutchison Chi-Med during the conduct of the study. The authors have no other conflicts of interest to declare.

Etbical Statement: The authors are accountable for all aspects of the work in ensuring that questions related to the accuracy or integrity of any part of the work are appropriately investigated and resolved. The study was conducted in accordance with the Declaration of Helsinki (as revised in 2013) and Guidelines for Good Clinical Practice, independently approved by the ethics committees of each participating center (NCT02976116) and informed consent was taken from all the patients.

Open Access Statement: This is an Open Access article distributed in accordance with the Creative Commons Attribution-NonCommercial-NoDerivs 4.0 International License (CC BY-NC-ND 4.0), which permits the noncommercial replication and distribution of the article with the strict proviso that no changes or edits are made and the original work is properly cited (including links to both the formal publication through the relevant DOI and the license). See: https://creativecommons.org/licenses/by-nc-nd/4.0/.

\section{References}

1. Bray F, Ferlay J, Soerjomataram I, et al. Global cancer statistics 2018: GLOBOCAN estimates of incidence and mortality worldwide for 36 cancers in 185 countries. CA Cancer J Clin 2018;68:394-424.

2. Feng RM, Zong YN, Cao S-M, et al. Current cancer situation in China: good or bad news from the 2018 Global Cancer Statistics? Cancer Commun (Lond) 2019;39:22 .

3. Li Z, Bian Y, Zeng L, et al. Life Expectancy, Causes of Death, Risk Factors in China and the U.S. Ann Glob Health 2017;83:407-14.

4. Travis WD, Brambilla E, Noguchi M, et al. International association for the study of lung cancer/american thoracic society/european respiratory society international multidisciplinary classification of lung adenocarcinoma. J Thorac Oncol 2011;6:244-85.

5. Hicklin DJ, Ellis LM. Role of the vascular endothelial growth factor pathway in tumor growth and angiogenesis. J Clin Oncol 2005;23:1011-27.

6. Sandler A, Gray R, Perry MC, et al. Paclitaxel-carboplatin alone or with bevacizumab for non-small-cell lung cancer. 
N Engl J Med 2006;355:2542-50.

7. Zhou C, Wu YL, Chen G, et al. BEYOND: A Randomized, Double-Blind, Placebo-Controlled, Multicenter, Phase III Study of First-Line Carboplatin/ Paclitaxel Plus Bevacizumab or Placebo in Chinese Patients With Advanced or Recurrent Nonsquamous NonSmall-Cell Lung Cancer. J Clin Oncol 2015;33:2197-204.

8. Garon EB, Ciuleanu TE, Arrieta O, et al. Ramucirumab plus docetaxel versus placebo plus docetaxel for secondline treatment of stage IV non-small-cell lung cancer after disease progression on platinum-based therapy (REVEL): a multicentre, double-blind, randomised phase 3 trial. Lancet 2014;384:665-73.

9. Reck M, Kaiser R, Mellemgaard A, et al. Docetaxel plus nintedanib versus docetaxel plus placebo in patients with previously treated non-small-cell lung cancer (LUMELung 1): a phase 3, double-blind, randomised controlled trial. Lancet Oncol 2014;15:143-55.

10. Zhang Y, Zou JY, Wang Z, et al. Fruquintinib: a novel antivascular endothelial growth factor receptor tyrosine kinase inhibitor for the treatment of metastatic colorectal cancer. Cancer Manag Res 2019;11:7787-803.

11. Lu S, Chang J, Liu X, et al. Randomized, Double-Blind, Placebo-Controlled, Multicenter Phase II Study of Fruquintinib After Two Prior Chemotherapy Regimens in Chinese Patients With Advanced Nonsquamous NonSmall-Cell Lung Cancer. J Clin Oncol 2018;36:1207-17.

12. Lu S, Chen G, Sun Y, et al. A Phase III, randomized, double-blind, placebo-controlled, multicenter study of fruquintinib in Chinese patients with advanced nonsquamous non-small-cell lung cancer - The FALUCA study. Lung Cancer 2020;146:252-62.

13. Mao L, Zhao W, Li X, et al. Mutation spectrum of EGFR in 21,324 patients with non-small cell lung cancer successfully tested by multiple platforms in a CAPaccredited laboratory. J Clin Oncol 2020;38:e13637.

14. Zhang WQ, Li T, Li H. Efficacy of EGFR tyrosine kinase inhibitors in non-small-cell lung cancer patients with/ without EGFR-mutation: evidence based on recent phase III randomized trials. Med Sci Monit 2014;20:2666-76.

15. Oizumi S, Kobayashi K, Inoue A, et al. Quality of life with gefitinib in patients with EGFR-mutated non-small cell lung cancer: quality of life analysis of North East Japan Study Group 002 Trial. Oncologist 2012;17:863-70.

16. Stewart EL, Tan SZ, Liu G, et al. Known and putative mechanisms of resistance to EGFR targeted therapies in NSCLC patients with EGFR mutations-a review. Transl Lung Cancer Res 2015;4:67-81.
17. Sonoda T, Nishikawa S, Sakakibara R, et al. EGFR T790M mutation after chemotherapy for small cell lung cancer transformation of EGFR-positive non-small cell lung cancer. Respir Med Case Rep 2018;24:19-21.

18. Cross DA, Ashton SE, Ghiorghiu S, et al. AZD9291, an irreversible EGFR TKI, overcomes T790M-mediated resistance to EGFR inhibitors in lung cancer. Cancer Discov 2014;4:1046-61.

19. Jänne PA, Yang JC, Kim DW, et al. AZD9291 in EGFR inhibitor-resistant non-small-cell lung cancer. N Engl J Med 2015;372:1689-99.

20. Mok TS, Wu YL, Ahn MJ, et al. Osimertinib or PlatinumPemetrexed in EGFR T790M-Positive Lung Cancer. N Engl J Med 2017;376:629-40.

21. Accestra [Internet]. Hangzhou: The Association; c2019 [cited 2020 Apr 26]. China New Drug Application (NDA) Approvals in Q1 2020; [about 2 screens]. Available online: https://www.accestra.com/china-new-drug-applicationnda-approvals-in-q1-2020/

22. Naumov GN, Nilsson MB, Cascone T, et al. Combined vascular endothelial growth factor receptor and epidermal growth factor receptor (EGFR) blockade inhibits tumor growth in xenograft models of EGFR inhibitor resistance. Clin Cancer Res 2009;15:3484-94.

23. Ren Y, Long J, Fan S, et al. Abstract 2089: Evaluation of fruquintinib, a potent and selective oral VEGFR inhibitor, in combination with targeted therapies or immune checkpoint inhibitors in preclinical tumor models. Cancer Res 2017;77:Abstract 2089.

24. Zhao Y, Wang H, Shi Y, et al. Comparative effectiveness of combined therapy inhibiting EGFR and VEGF pathways in patients with advanced non-small-cell lung cancer: a meta-analysis of 16 phase II/III randomized trials. Oncotarget 2017;8:7014-24.

25. Saito H, Fukuhara T, Furuya N, et al. Erlotinib plus bevacizumab versus erlotinib alone in patients with EGFR-positive advanced non-squamous non-small-cell lung cancer (NEJ026): interim analysis of an open-label, randomised, multicentre, phase 3 trial. Lancet Oncol 2019;20:625-35.

26. Nakagawa K, Garon EB, Seto T, et al. Ramucirumab plus erlotinib in patients with untreated, EGFRmutated, advanced non-small-cell lung cancer (RELAY): a randomised, double-blind, placebo-controlled, phase 3 trial. Lancet Oncol 2019;20:1655-69.

27. FDA [Internet]. Maryland: The Administration; [cited 2020 Jun 01]. FDA approves ramucirumab plus erlotinib for first-line metastatic NSCLC 2020; [about 2 screens]. 
Available online: https://www.fda.gov/drugs/drugapprovals-and-databases/fda-approves-ramucirumab-pluserlotinib-first-line-metastatic-nsclc

28. Ichihara E, Hotta K, Nogami N, et al. Phase II trial of gefitinib in combination with bevacizumab as firstline therapy for advanced non-small cell lung cancer with activating EGFR gene mutations: the Okayama Lung Cancer Study Group Trial 1001. J Thorac Oncol 2015;10:486-91.

29. Maemondo M, Inoue A, Kobayashi K, et al. Gefitinib or chemotherapy for non-small-cell lung cancer with mutated EGFR. N Engl J Med 2010;362:2380-8.

30. Gijtenbeek RG, Damhuis RA, Groen HJ, et al. Nationwide Real-world Cohort Study of First-line Tyrosine Kinase Inhibitor Treatment in Epidermal Growth Factor Receptor-mutated Non-small-cell Lung Cancer. Clin Lung Cancer 2020;21:e647-e653.

31. Okamoto I, Morita S, Tashiro N, et al. Real world treatment and outcomes in EGFR mutation-positive nonsmall cell lung cancer: Long-term follow-up of a large patient cohort. Lung Cancer 2018;117:14-9.

32. Lau SC, Chooback N, Ho C, et al. Outcome Differences Between First- and Second-generation EGFR Inhibitors in Advanced EGFR Mutated NSCLC in a Large Population-based Cohort. Clin Lung Cancer 2019;20:e576-e583.

33. Patel N, Wu P, Zhang H. Comparison of gefitinib as first- and second-line therapy for advanced lung adenocarcinoma patients with positive exon 21 or 19 del epidermal growth factor receptor mutation. Cancer Manag Res 2017;9:243-8.

34. Li XN, Qiu D, Pan X, et al. Mutation of the epidermal growth factor receptor gene and its impact on the efficacy of gefitinib in advanced non-small cell lung cance. Int J
Clin Exp Med 2015;8:5397-405.

35. Kim DW, Lee SH, Lee JS, et al. A multicenter phase II study to evaluate the efficacy and safety of gefitinib as first-line treatment for Korean patients with advanced pulmonary adenocarcinoma harboring EGFR mutations. Lung Cancer 2011;71:65-9.

36. Sim EH, Yang IA, Wood-Baker R, et al. Gefitinib for advanced non-small cell lung cancer. Cochrane Database Syst Rev 2018;1:CD006847.

37. Fukuoka M, Wu YL, Thongprasert S, et al. Biomarker analyses and final overall survival results from a phase III, randomized, open-label, first-line study of gefitinib versus carboplatin/paclitaxel in clinically selected patients with advanced non-small-cell lung cancer in Asia (IPASS). J Clin Oncol 2011;29:2866-74.

38. Zeng L, Xiao L, Jiang W, et al. Investigation of efficacy and acquired resistance for EGFR-TKI plus bevacizumab as first-line treatment in patients with EGFR sensitive mutant non-small cell lung cancer in a Real world population. Lung Cancer 2020;141:82-8.

39. Yu HA, Arcila ME, Hellmann MD, et al. Poor response to erlotinib in patients with tumors containing baseline EGFR T790M mutations found by routine clinical molecular testing. Ann Oncol 2014;25:423-8.

40. Jenkins S, Yang JC, Ramalingam SS, et al. Plasma ctDNA Analysis for Detection of the EGFR T790M Mutation in Patients with Advanced Non-Small Cell Lung Cancer. J Thorac Oncol 2017;12:1061-70.

41. Soria JC, Ohe Y, Vansteenkiste J, et al. Osimertinib in Untreated EGFR-Mutated Advanced Non-Small-Cell Lung Cancer. N Engl J Med 2018;378:113-25.

42. Tan CS, Kumarakulasinghe NB, Huang YQ, et al. Third generation EGFR TKIs: current data and future directions. Mol Cancer 2018;17:29.
Cite this article as: Lu S, Zhou JY, Niu XM, Zhou JY, Jian H, Yin HY, Guan S, Wang LF, Li K, He J, Su WG. Fruquintinib with gefitinib as first-line therapy in patients carrying EGFR mutations with advanced non-small cell lung cancer: a single-arm, phase II study. Transl Lung Cancer Res 2021;10(2):839-854. doi: 10.21037/tlcr-20-1028 


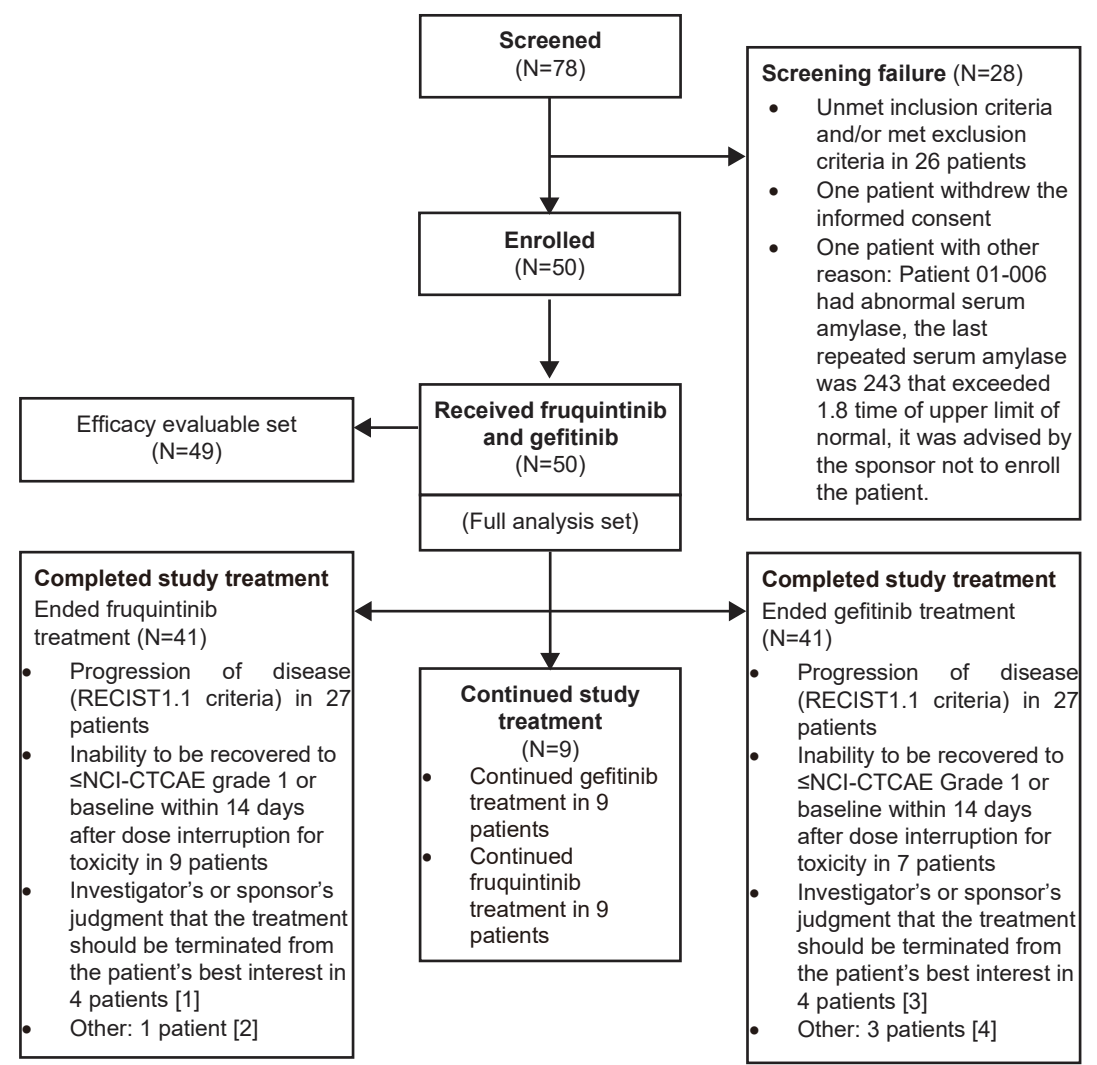

Figure S1 CONSORT diagram. Patient disposition through 28 June 2019. [1], the reason for termination of treatment was recorded as investigator's or sponsor's judgment that the treatment should be terminated for the patient's best interest. Patient 01-014 (date of termination 10 October 2017) experienced a bronchopleural fistula on 9 October 2017, leading to discontinuation of fruquintinib and gefitinib. [2], Patient 02-017 withdrew from the treatment voluntarily after consideration. [3], the reason for termination of treatment was recorded as investigator's or sponsor's judgment that the treatment should be terminated for the patient's best interest. [4], the reason for termination of treatment was recorded as 'other' in Patient 02-013 [voluntary withdrawal for repeated drug-induced liver injury (date of termination 6 February 2018)]; however, the adverse event was recorded as increased glutamic-pyruvate transaminase on October 31 , 2017, leading to discontinuation of gefitinib. The reason for termination of treatment was recorded as 'other' in Patient 02-014 (permanent discontinuation of gefitinib for interstitial pneumonia: date of termination 9 April 2018). NCI-CTCAE, National Cancer Institute Common Terminology Criteria for Adverse Events. Patient 02-017 withdrew from the treatment voluntarily after consideration. 
Table S1 Inclusion and exclusion criteria

Inclusion criteria (all the following conditions had to be met prior to enrollment)

1. Patients understood the study protocol adequately, and voluntarily signed the informed consent form

2. Patients were with histologically and/or cytologically confirmed stage IIIB/IV non-squamous non-small-cell lung cancer (NSCLC) and naïve to the systematic treatment for advanced diseases

3. Presence of epidermal growth factor receptor (EGFR)-sensitive mutation (EGFR exon-19 deletion or exon-21 L858R mutation)

4. Presence of at least one measurable tumor lesion in accordance with RECIST 1.1 criteria [having not received radiotherapy for the lesion, major axis of the lesion $\geq 10 \mathrm{~mm}$ in CT or MRI during baseline screening (minor axis $\geq 15 \mathrm{~mm}$ for lymph node lesion)]

5. Aged $18-75$ years

6. Physical performance score (Eastern Cooperative Oncology Group performance status score) 0-1

7. Expected survival $>3$ months

Exclusion criteria (subjects were excluded from this study if any one of the following criteria were identified)

1. Prior systematic treatment for advanced lung cancer (e.g., chemotherapy, targeted therapy); participation in and administration of the drug in other clinical trials within 4 weeks prior to the first dose of the investigational product; having received biotherapy or immunotherapy within 4 weeks prior to the study treatment; having received the traditional Chinese medicine with antitumor activity within 1 week prior to the study treatment

2. Recurrence or metastasis occurred during or within 1 year after completion of neoadjuvant/adjuvant chemotherapy (if applicable)

3. Received palliative radiotherapy within 1 week prior to the study treatment; proportion of bone marrow $>30 \%$ at the site receiving radiotherapy within 4 weeks prior to study treatment, or having received radical/extensive radiation therapy (if applicable); brachytherapy within 60 days prior to the first dose of investigational product (e.g., particle implantation)

4. Prior treatment with tyrosine kinase inhibitor (TKI) or monoclonal antibody against EGFR

5. Prior treatment with TKI or monoclonal antibody against vascular endothelial growth factor (VEGF) and/or VEGF receptor

6. Known T790M mutation

7. Received potent inhibitor and/or inducer of cytochrome P450 3A4 (CYP3A4) within 2 weeks prior to the treatment of investigational product

8. Received P-glycoprotein (P-gp) and breast cancer resistance protein (BCRP) substrate within 2 weeks prior to the treatment of investigational product

9. Major surgery or large invasive procedure within 60 days prior to the first dose of investigational product; or incomplete recovery from prior surgery/procedure (if applicable)

10.Presence of active brain metastasis prior to the study (no prior radiation to brain or stable of clinical symptoms $<4$ weeks after brain radiotherapy or having clinical symptoms)

11.Radiological evidence showing tumor invasion to or encompassing major vessels of lungs (e.g., pulmonary artery, superior vena cava)

12.Prior interstitial lung disease, or drug-induced interstitial disease, or radiation pneumonitis requiring hormone therapy, or any active interstitial lung disease with clinical evidence; pulmonary insufficiency requiring oxygen inhalation (judgment criteria: clinical examination or arterial partial pressure of oxygen $<70 \mathrm{mmHg}$

13.Dysphagia or known drug absorption disorder; refractory nausea/vomiting; any serious disease or infection affecting absorption of investigational product, for example, environment-related frequent diarrhea

14.Serious ocular disease ( $\geq$ CTCAE grade 2), including but not limited to ulcerative conjunctivitis, ulcerative keratitis, corneal erosion

15. Clinically uncontrolled active infection, including but not limited to acute pneumonia

16. Known history of significant hepatic disease, including but not limited to hepatitis $B$ virus (HBV) infection and positive HBV DNA $\left(\geq 1 \times 10^{4}\right.$ $/ \mathrm{mL}$ or $\geq 2,000 \mathrm{IU} / \mathrm{mL}$ ); known hepatitis $\mathrm{C}$ virus (HCV) infection and positive HCV RNA, or liver cirrhosis

17.Known HIV infection

Table S1 (continued) 
Table S1 (continued)

18.Currently active duodenal ulcer, ulcerative colitis, intestinal obstruction or other conditions as judged by investigators to possibly cause gastrointestinal hemorrhage or perforation; prior history of intestinal perforation or fistula

19.Other malignant tumors in the past 5 years, except the basal cell carcinoma of skin or cervical carcinoma in situ that has received curative therapy

20.Subjects will be excluded for the following conditions

a. Absolute neutrophil count $9 / \mathrm{L}$, or platelet $9 / \mathrm{L}$, or hemoglobin $<9 \mathrm{~g} / \mathrm{dL}$ in the laboratory examination within 1 week prior to the first dose of investigational product

s. Serum total bilirubin exceeding upper limits of normal (ULN), or alanine aminotransaminase (ALT) or aspartate aminotransaminase (AST) exceeding 1.5 times the ULN (based on the normal value at the clinical study center); ALT or AST exceeding 3 times the ULN for the patients with hepatic metastasis

d. Presence of clinically significant electrolyte abnormality

f. Serum creatinine exceeding 1.5 times the ULN (based on the normal value at the clinical study center) or creatinine clearance lower than $60 \mathrm{~mL} / \mathrm{min}$

g. Calculation formula for creatinine clearance rate $(\mathrm{Ccr})=(140-$ age $) \times$ weight $(\mathrm{kg}) /[72 \times \mathrm{Scr}(\mathrm{mg} / \mathrm{dll})]$, or Ccr $=[(140-\mathrm{age}) \times$ weight $(\mathrm{kg})]$ $\times 1.23 /[\mathrm{Scr}(\mu \mathrm{mol} / \mathrm{L})]$, the unit of creatinine should be noted in the calculation of Ccr, the result calculated $\times 0.85$ for women

h. Urine protein $2+$ or above, or 24 -hour urine protein quantitation $\geq 1.0 \mathrm{~g} / 24 \mathrm{~h}$

j. Activated partial thromboplastin time exceeding 1.5 times the ULN (based on the normal value at the clinical study center) or international normalized ratio $>1.5$

h. Uncontrollable hypertension, i.e., systolic blood pressure $\geq 140 \mathrm{mmHg}$ and/or diastolic blood pressure $\geq 90 \mathrm{mmHg}$ after drug therapy

i. Cardiac function assessment: left ventricular ejection fraction $<50 \%$ (by echocardiogram) moderate or above mitral or tricuspid insufficiency

j. Acute myocardial infarction, serious/unstable angina pectoris or coronary artery bypass grafting within 6 months prior to the first dose of investigational product; or New York Heart Association grade 2 or above cardiac insufficiency

k. Stroke event and/or transient ischemic attack within 12 months prior to the first dose of investigational product

I. Mean QT interval (QTC) corrected using Fridericia formula $>470 \mathrm{msec}$ on 12-lead electrocardiogram (ECG) (at least two measurements); calculation formula: QTcF = QTc (Fridericia) $=\mathrm{QT} /(\mathrm{RR} / 1000) 1 / 3$

21. History of arterial thrombosis or deep venous thrombosis within 6 months prior to the first dose of investigational product, or evidence or history of thrombotic or hemorrhagic tendency within 2 months prior to enrollment (e.g., $\geq 3 \mathrm{~cm}$ hepatic hemangioma), regardless of severity; history of hemoptysis (defined as blood loss $>2.5 \mathrm{~mL}$, and blood appearing bright red) within 1 month prior to enrollment

22. Current (within 10 days prior to the first dose of investigational product) use of thrombolytic therapy or therapeutic use of anticoagulant (except preventive use of anticoagulants)

23. Incomplete recovery of serious ulcer or fracture at skin wound, site of trauma or mucosa

24.Pregnant or lactating women or positive pregnancy test for females of childbearing potential prior to the first dose (if applicable)

25.Patients who have childing-bearing potential but are not willing to use contraceptive measures, or whose sex partners who are not willing to use contraceptive measures

26. Presence of any clinical or laboratory abnormality regarded inappropriate to participate in this clinical study, as considered by investigators

27.Presence of serious psychological or mental abnormality, poor compliance with participation in this clinical study as evaluated 28.Serious allergic reaction to the active substance of fruquintinib and/or gefitinib and/or any excipient in the investigational product

CT, computerized tomography; MRI, magnetic resonance imaging CTCAE, National Cancer Institute Common Terminology Criteria for Adverse Events. 
Table S2 Exposure to investigational treatment (FAS)

\begin{tabular}{|c|c|c|c|c|c|c|}
\hline Parameters & \multicolumn{2}{|c|}{$\begin{array}{l}\text { Fruquintinib [4 mg QD }\left(\text { Cycle }^{\dagger}\right) \\
\text { then } 5 \mathrm{mg} \text { ] + Gefitinib } 250 \mathrm{mg} \\
\text { QD }(\mathrm{N}=26)\end{array}$} & \multicolumn{2}{|c|}{$\begin{array}{l}\text { Fruquintinib (4 mg QD) + Gefitinib } \\
\qquad 250 \mathrm{mg} \text { QD }(\mathrm{N}=24)\end{array}$} & \multicolumn{2}{|c|}{ Total $(\mathrm{N}=50)$} \\
\hline $\begin{array}{l}\text { Median duration of exposure } \\
(\text { months })^{\ddagger}\end{array}$ & 12.8 & 12.8 & 13.3 & 13.3 & 12.9 & 12.9 \\
\hline Mean RDI ${ }^{\S}, \mathrm{n}(\%)$ & $0.78(0.183)$ & $0.96(0.075)$ & $0.82(0.153)$ & $0.97(0.042)$ & $0.80(0.168)$ & $0.97(0.061)$ \\
\hline
\end{tabular}

Table S3 Summary of AEs leading to discontinuation and dose adjustment, SAEs and AEs leading to death—FAS

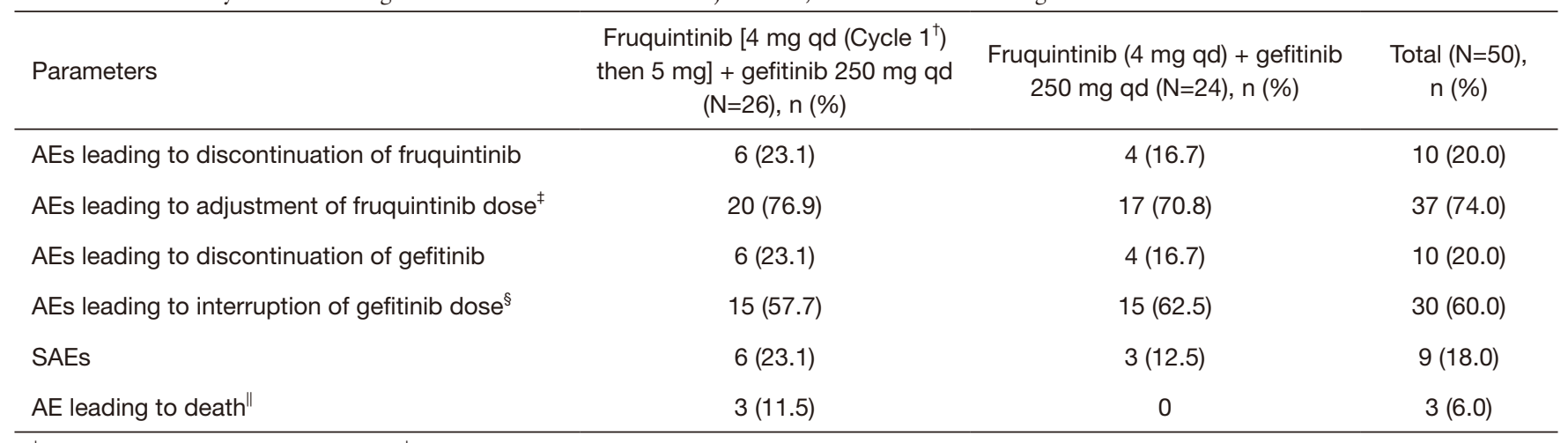

${ }^{\dagger}$, Cycle 1 was 4 weeks duration; ${ }^{\ddagger}$, dose adjustment included dose interruption and reduction. The adverse events (AEs) leading to dose adjustment did not include the AEs leading to discontinuation of the study treatments however, dose interruption for an AE was recorded in the drug record form for the four patients; ${ }^{\S}$, the AEs leading to dose interruption did not include the AEs leading to discontinuation of the study treatments however, dose interruption for an AE was recorded in the drug record form for the four patients; ", patient 01013 received treatment for 257 days, and died ( $A E$ term: acute cerebral infarction) 20 days after the last dose of study treatment; Patient 01-014 received the treatment for 109 days, and died (AE term: death) from progression of disease 26 days after the last dose of study treatment; Patient 01-024 received the treatment for 104 days, and died (AE term: death) of unknown cause 19 days after the last dose of study treatment. Investigators concluded that three deaths as possibly unrelated to fruquintinib and gefitinib. AE, adverse event; qd, once daily; SAE, serious adverse event. 
Table S4 Adverse events of special interest (incidence in $\geq 20 \%$ of all patients) summarized by preferred term-FAS

\begin{tabular}{|c|c|c|c|c|c|c|}
\hline \multirow[t]{2}{*}{ AESI category/preferred term } & \multicolumn{2}{|c|}{$\begin{array}{l}\left.\text { Fruquintinib [4 mg qd (Cycle } 1^{\dagger}\right) \text { then } \\
5 \mathrm{mg} \text { ] + gefitinib qd }(\mathrm{N}=26), \mathrm{n}(\%)\end{array}$} & \multicolumn{2}{|c|}{$\begin{array}{l}\text { Fruquintinib (4 mg qd) + gefitinib } \\
\qquad q d(N=24), n(\%)\end{array}$} & \multicolumn{2}{|c|}{$\begin{array}{l}\text { Total }(\mathrm{N}=50) \\
\mathrm{n}(\%)\end{array}$} \\
\hline & All grades & Grade $\geq 3$ & All grades & Grade $\geq 3$ & All grades & Grade $\geq 3$ \\
\hline Hepatotoxicity ${ }^{\S}$ & $25(96.2)$ & $10(38.5)$ & $20(83.3)$ & $3(12.5)$ & $45(90.0)$ & $13(26.0)$ \\
\hline Aspartate aminotransferase increased & $23(88.5)$ & $4(15.4)$ & 19 (79.2) & $1(4.2)$ & $42(84.0)$ & $5(10.0)$ \\
\hline Bilirubin conjugated increased & $14(53.8)$ & $1(3.8)$ & $8(33.3)$ & $1(4.2)$ & $22(44.0)$ & $2(4.0)$ \\
\hline Blood bilirubin increased & $10(38.5)$ & 0 & $5(20.8)$ & 0 & $15(30.0)$ & 0 \\
\hline Gamma-glutamyltransferase increased & $5(19.2)$ & $2(7.7)$ & $7(29.2)$ & $1(4.2)$ & $12(24.0)$ & $3(6.0)$ \\
\hline Hyperthyroidism & $8(30.8)$ & 0 & $10(41.7)$ & 0 & $18(36.0)$ & 0 \\
\hline Proteinuria & $18(69.2)$ & $3(11.5)$ & $20(83.3)$ & $1(4.2)$ & $38(76.0)$ & $4(8.0)$ \\
\hline Proteinuria & $18(69.2)$ & $3(11.5)$ & $20(83.3)$ & $1(4.2)$ & $38(76.0)$ & $4(8.0)$ \\
\hline Skin toxicity & $19(73.1)$ & $1(3.8)$ & $14(58.3)$ & $3(12.5)$ & $33(66.0)$ & $4(8.0)$ \\
\hline $\begin{array}{l}\text { Palmar-plantar erythrodysesthesia } \\
\text { syndrome }\end{array}$ & $11(42.3)$ & 0 & $6(25.0)$ & 0 & $17(34.0)$ & 0 \\
\hline Dermatitis acneiform & $8(30.8)$ & 0 & $6(25.0)$ & 0 & $14(28.0)$ & 0 \\
\hline Rash & $5(19.2)$ & 0 & $5(20.8)$ & $1(4.2)$ & $10(20.0)$ & $1(2.0)$ \\
\hline
\end{tabular}

${ }^{\dagger}$, Cycle 1 was 4 weeks duration; ${ }^{\ddagger}$, adverse event of special interest (AESIs) specified in the protocol were determined in accordance with standardized MedDRA Query (SMQ) terms. In case the same adverse event occurred multiple times for the same patient, the event could only be counted once in calculation by the same AESI category and preferred term for the patient; ${ }^{\S}$, hepatotoxicity was defined as transaminase abnormal, hepatic function abnormal or liver injury. FAS, full analysis set; qd, once daily. 


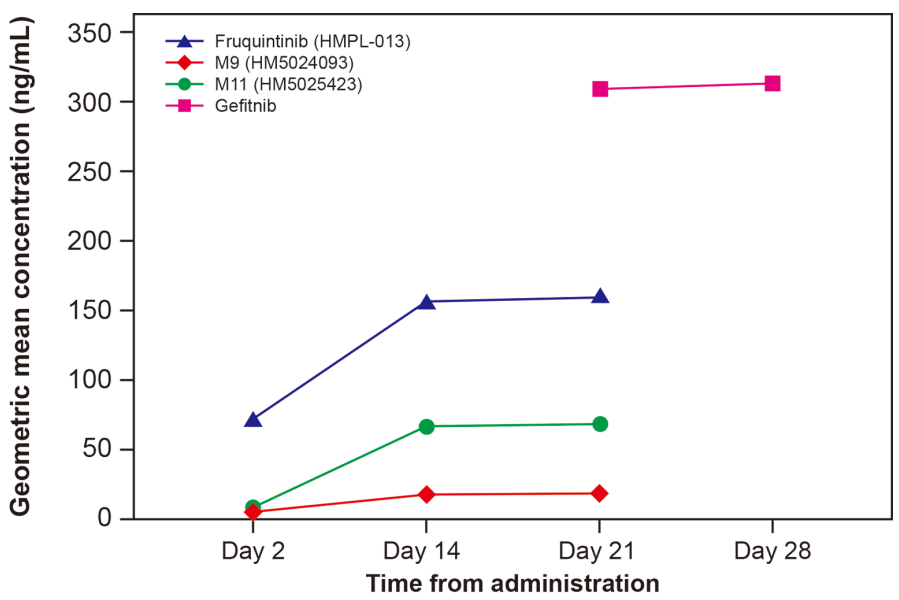

Figure S2 Geometric mean plasma concentrations $(\mathrm{ng} / \mathrm{mL})$ of study treatments over Cycle 1. Mean plasma concentrations of fruquintinib (HMPL-013) (4 mg), and its metabolites M11 (HM5025423) and M9 (HM5024093), taken at Day 2, 14, and 21 of Cycle 1. Mean plasma concentrations of gefitinib $(250 \mathrm{mg})$ taken at Day 21 and 28 of Cycle 1. 